\title{
Aprendizaje-servicio aplicado a la dirección estratégica de asociaciones sin fin de lucro
}

\author{
Calvo-Babío, Nuria'; Barros-Campello, Esther²; Gago-Cortés, Carmen² \\ ${ }^{1}$ Universidade da Coruña, Facultad de Economía y Empresa, \\ orcid.org/0000-0002-5711-5015 \\ ²Universidade da Coruña, Facultad de Economía y Empresa,
}

\section{RESUMEN}

Proponemos un planteamiento orientado al aprendizaje basado en el servicio a organizaciones sin fin de lucro del entorno de la Universidade da Coruña (UDC). Para ello, y utilizando como punto de partida las competencias establecidas en el plan de estudios de grado en Dirección y Administración de Empresas, se presenta un esquema de la dinámica del proceso de enseñanza-aprendizaje que, incorporando aspectos relacionados con el contexto educativo y la propia motivación del estudiante, permite una planificación de tareas orientada al desarrollo de competencias profesionales por un lado, y a la entrega de informes de análisis estratégico a los responsables de las asociaciones por otro. El objetivo de esta experiencia es el de difundir comportamientos de responsabilidad social corporativa entre los estudiantes universitarios en el marco de su proceso de aprendizaje. Por ello, los docentes de esta asignatura planteamos trabajar con los estudiantes la formulación estratégica de organizaciones sin fin de lucro que previamente habían firmado un convenio con la UDC y ofrecer los informes generados a los responsables de las organizaciones implicadas.

PALABRAS CLAVE: método del caso; dirección estratégica; aprendizaje-servicio 


\section{CITA RECOMENDADA:}

Calvo-Babío, Nuria; Barros-Campello, Esther; Gago-Cortés, Carmen (2020): Aprendizaje-servicio aplicado a la dirección estratégica de asociaciones sin fin de lucro. En De la Torre Fernández, E. (ed.) (2020). Contextos universitarios transformadores: Boas prácticas no marco dos GID. IV Xornadas de Innovación Docente. Cufie. Universidade da Coruña. A Coruña (págs. 281-294).

DOI capítulo: https://doi.org/10.17979/spudc.9788497497756.281

DOI libro: https://doi.org/10.17979/spudc. 9788497497756

\section{ABSTRACT}

We propose a service-based learning focused on non-profit organizations connected to the University of A Coruña (UDC). We used the competences-based framework of the degree in Business Management and Administration of the University of A Coruña, and proposed a teaching-learning process involving the educational context and the student's own motivation. We also elaborated a planning of tasks that were oriented to the development of professional competences on the one hand, and the delivery of strategic analysis reports for non-profit associations on the other. Therefore, the teachers of this subject proposed to work with students the strategic formulation of nonprofit organizations, which had a previous agreement with the University of A Coruña, and offer the reports to the representatives of the organizations involved.

KEY WORDS: case study, strategic management, service-based learning 


\section{INTRODUCCIÓN}

El objetivo descrito en el Plan de Grado en Administración y Dirección de Empresas de la Universidad de A Coruña es el de formar profesionales capaces de desempeñar labores de gestión, asesoramiento y evaluación en las organizaciones empresariales. Para ello, el graduado deberá manejar los conceptos y técnicas empleados en las diferentes áreas funcionales de la empresa, entender las relaciones que existen entre ellas y con los objetivos de la organización; tendrá que ser capaz de tomar decisiones y, en general, de asumir tareas directivas (Universidade da Coruña, 2008).

En la distribución de módulos establecida en el Plan de Grado, el módulo de Organización de Empresas plantea contribuir al desarrollo de la mayor parte de las competencias propuestas para el conjunto de la titulación. Dado que el área de Organización de Empresas persigue analizar la problemática de la empresa como una organización que busca alcanzar sus objetivos, resulta especialmente relevante que los futuros profesionales que trabajen en esta disciplina dispongan de las competencias específicas y transversales previstas en el módulo temático.

El programa de Dirección Estratégica y política empresarial I está diseñado para ser impartida en el tercer curso del Plan de Grado en Administración y Dirección de Empresas (en inglés y castellano) y en cuarto curso del Doble Grado en Derecho y Administración y Dirección de Empresas. Esta asignatura es también el primer contacto en profundidad con la dirección de empresas en general y, más en concreto con el estudio de la dirección estratégica y las políticas de empresa. Con este programa se pretende alcanzar un objetivo articulado en cuatro perspectivas:

1. La primera conceptual, proporcionará a los estudiantes el marco teórico necesario para abordar los problemas de dirección de empresas.

2. La segunda instrumental, proporcionará a los estudiantes una serie de herramientas de análisis estratégico disponibles y relacionadas con el marco teórico presentado. 
3. La tercera de enfoque. Dada la multitud de enfoques y aproximaciones al campo de la dirección estratégica y, conscientes de la formación de los alumnos de las facultades de ciencias económicas, se enfatiza la aplicación de los conceptos económicos para el desarrollo del análisis estratégico.

4. La cuarta pragmática. La naturaleza de la asignatura obliga a la referencia continua a casos. Por ello cada uno de los temas se irá ilustrando con diferentes casos extraídos de dos fuentes básicas: libros de casos y casos desarrollados por los docentes en su labor investigadora. La búsqueda de estos últimos ha de ser una tarea que descansará también en el trabajo del alumno fomentando así su capacidad de identificar cuestiones estratégicas en la vida económica cotidiana.

El estudio de casos permite analizar el fenómeno estudiado en su contexto real, utilizando múltiples fuentes de evidencia, cuantitativas y/o cualitativas simultáneamente, así como fuentes de información primaria y secundaria. También conlleva el empleo de abundante información subjetiva, la imposibilidad de aplicar la inferencia estadística y una elevada influencia del juicio subjetivo del investigador en la selección e interpretación de la información (Villarreal, 2011; Villarreal y Landeta, 2010; Yin, 1994).

La utilización del caso como instrumento educativo se está generalizando en la mayoría de los centros de formación en dirección de empresas. El estudio de casos constituye un elemento clave en el proceso de aprendizaje de los alumnos. En los últimos cursos de grado el análisis de casos puede servir como elemento integrador del conjunto de conocimientos adquirido por ellos, en las diversas disciplinas.

El estudiante se enfrenta a un problema 0 una serie de problemas relacionados que presenta el caso, aunque la mayoría de los casos no tienen solución única ni correcta, sino que hay varias posibilidades de resolución, como ocurre en la vida real de la empresa. El objetivo principal de este método es enseñar al estudiante cómo pensar y actuar ante distintas contingencias, dejando clara la importancia del análisis, frente a las conclusiones finales que, probablemente, 
serán múltiples. De este modo, los estudiantes se ven obligados a reflexionar sobre las posibilidades de aplicación de los conocimientos adquiridos en situaciones más abiertas y complejas que las de tipo estandarizado que se pueden plantear en las clases teóricas y prácticas de ejercicios y problemas. El método se fundamenta sobre el principio de la exposición múltiple. Para ello el caso debe estar bien planteado, lo que permitirá extraer de él toda su posible utilidad, huyendo de planteamientos ambiguos y superficiales. Las características básicas que debe reunir un caso son las siguientes:

- Una situación concreta producida en la realidad, nacida de la vida profesional.

- Un problema que exija un diagnóstico, una decisión, o ambas cosas.

- El suministro de una información suficiente que permita al alumno un enfoque correcto del problema.

- Una situación "total", en el sentido de que la información anterior más todas las eventuales informaciones suplementarias sean la base completa para el desarrollo del caso.

- Las generalizaciones posteriores sobre los resultados obtenidos.

El objetivo planteado por el Grupo de Innovación Educativa CABARO durante el curso 20182019 fue el de trabajar las competencias a desarrollar en la disciplina de Dirección Estratégica y Política Empresarial I en un proyecto de aprendizaje-servicio que, utilizando el método del caso como método de aprendizaje, permitiera a los alumnos acceder a una organización sin fin de lucro y desarrollar un análisis estratégico de la misma, aplicando sus conocimientos a una problemática empresarial real y ofreciendo al mismo tiempo el valor de su trabajo a los profesionales de estas organizaciones sin fin de lucro. 


\section{DESCRICIÓN DE LA EXPERIENCIA}

\subsection{TRABAJO PREVIO Y NUEVO ENFOQUE}

La aplicación del método del caso a la estrategia de enseñanza de la asignatura de Dirección estratégica y política empresarial I en el grado de Administración de Empresas, grado de Administración de Empresas bilingüe y doble grado en Derecho y Administración de empresas desde 2014 a 2018 nos ha permitido alinear las tres perspectivas implicadas en el proceso de enseñanza-aprendizaje:

- Visión de la institución: alineación de competencias (learning by doing)

- Visión del docente: Análisis multidisciplinar (aplicación de conceptos).

- Visión del estudiante: estudiar algo útil, entrar en la empresa y aportar valor.

Desde 2014 los docentes de la asignatura de Dirección Estratégica y Política Empresarial I hemos pedido a los alumnos que elaboren un caso de estudio de una empresa de su entorno a la que tengan acceso, preferiblemente pequeña o mediana empresa (PYME), sobre la que tienen que hacer un análisis estratégico, identificar su formulación estratégica y proponer mejoras y vectores de crecimiento. Para ello debían consultar previamente fuentes de información secundarias, contactar con la empresa y asegurarse de conseguir su compromiso de participación, entrevistar a diversas personas de la organización para asegurar la triangulación de la información y sacar conclusiones aplicando el marco conceptual estudiado en la asignatura.

Hasta noviembre de 2018, esta experiencia ha permitido documentar el análisis estratégico de 135 empresas-caso en el entorno local de los estudiantes de Administración de Empresas de la Universidade da Coruña.

La Figura 1 describe la evolución del número de empresas analizadas a lo largo de los cuatro cursos en los que se ha desarrollado esta experiencia. De las 135 empresas analizadas, 40 casos prácticos fueron realizados por los alumnos en el curso 2014/2015, 32 en el curso 2015/2016, 27 en el curso 2016/2017 y 36 en el curso 2017/2018. 


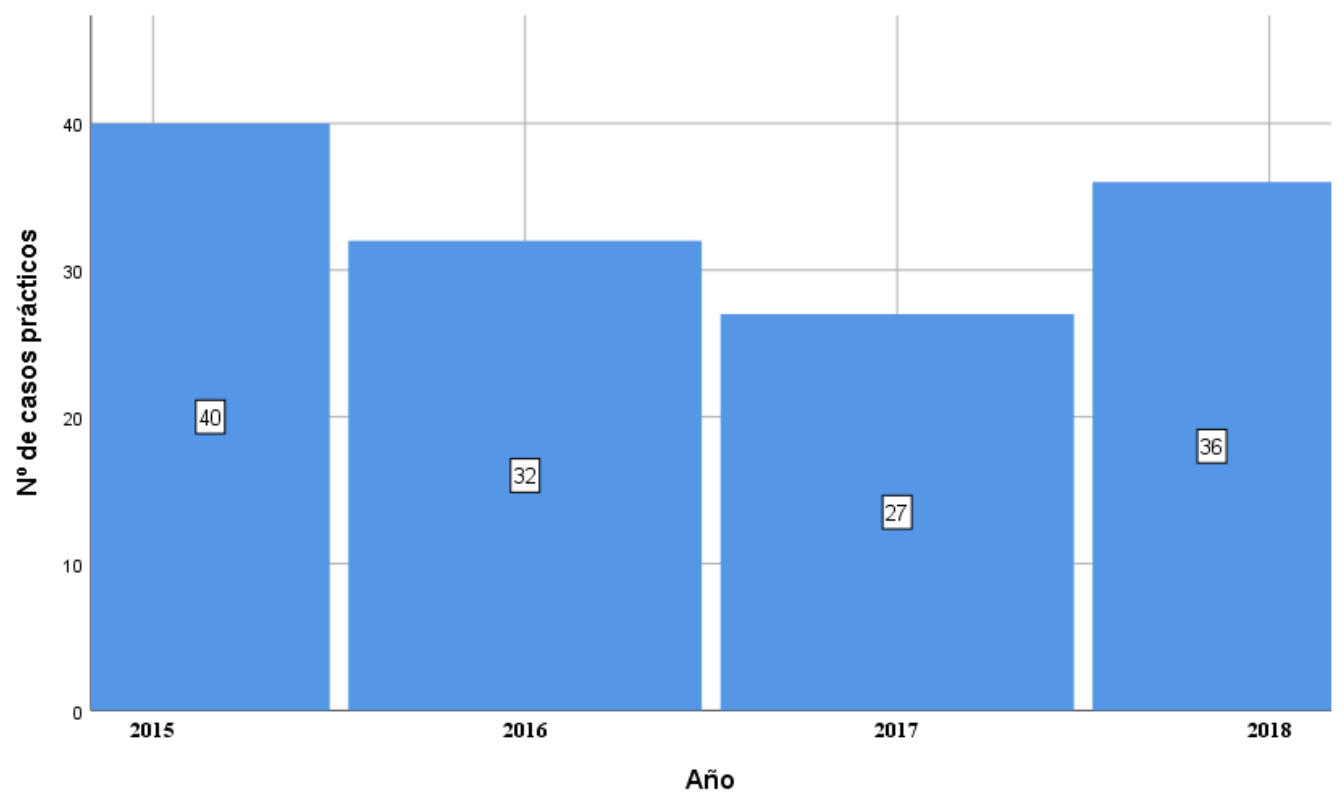

Figura 1: Número de casos prácticos realizados por curso

La Figura 2 nos da una visión de las empresas analizadas en los casos de estudio a través del sector de actividad en el que operan. La clasificación de las empresas se ha realizado a partir del código nacional de actividades económicas (CNAE) primario de cada una. Puede apreciarse que a través del análisis de casos se potencia el acercamiento de los estudiantes a la diversidad de realidad empresarial del entorno. De las 21 grandes categorías en las que se puede dividir la CNAE, 14 de ellas ya han sido analizadas a través de algún caso de estudio realizado por los estudiantes en estos primeros cuatro cursos de experiencia docente. Los sectores de actividad más analizados han sido las actividades comerciales, al por mayor y al por menor, con un $37 \%$ de los casos de estudio y las actividades de la industria manufacturera con un $18 \%$. Estos resultados parecen lógicos si tenemos en cuenta que estos dos grupos de sectores son los que concentran un mayor número de actividades de la clasificación CNAE y que además recogen de forma clara la estructura empresarial del entorno en que se mueven los estudiantes de la Universidade da Coruña. 


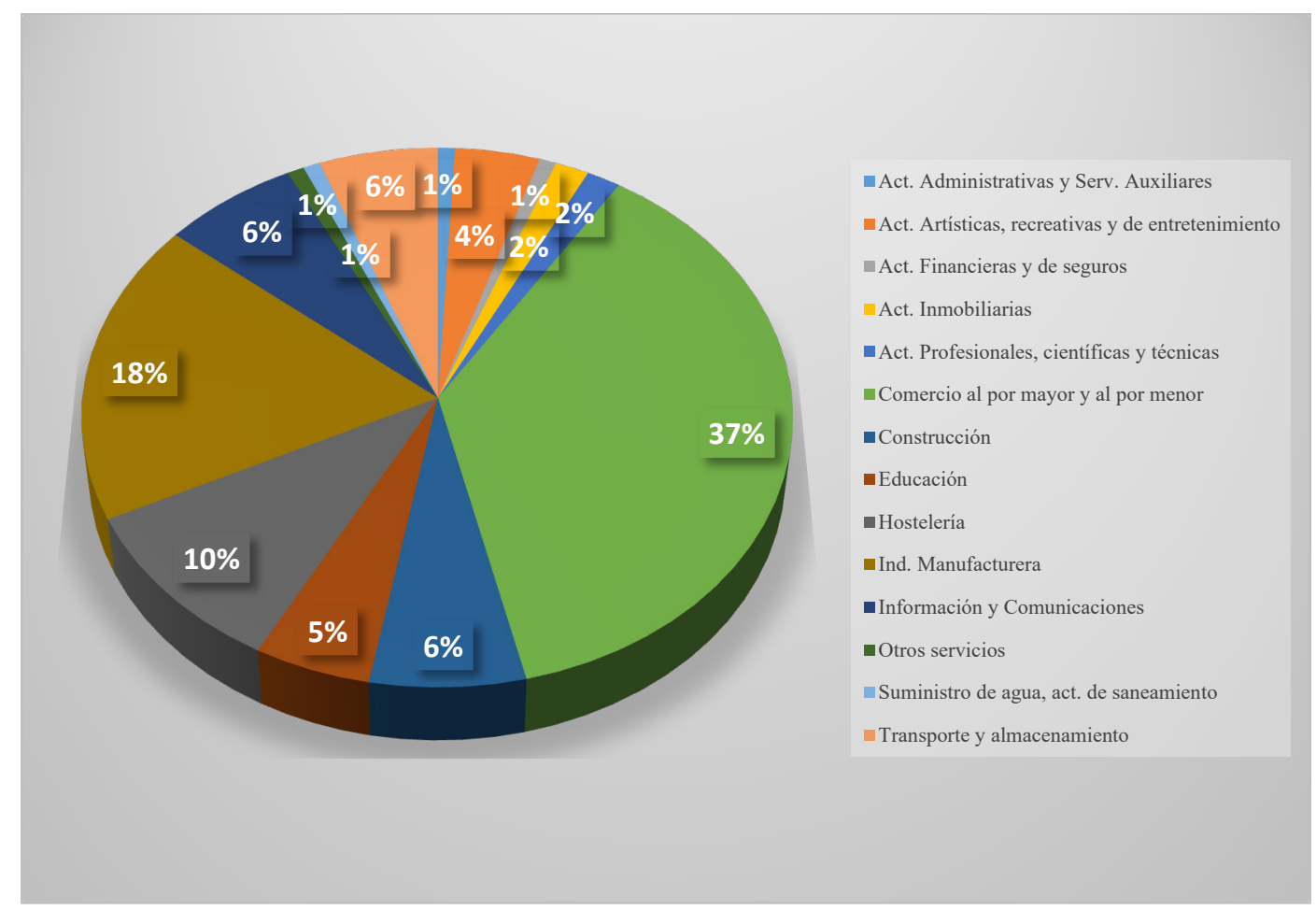

Figura 2. Distribución de los casos de estudio según la actividad de las empresas analizadas

Sin embargo, quedaba pendiente trabajar con organizaciones sin fin de lucro, caracterizadas por mantener estructuras organizativas poco profesionalizadas y basadas en el trabajo de voluntarios con una permanencia intermitente, escasa orientación a resultados y difícil supervivencia por su excesiva dependencia de subvenciones públicas.

Por ello, durante el curso académico 2019-2020, y de acuerdo a los objetivos planteados en el GIE CABAR0, se ofreció a los alumnos la posibilidad de trabajar con organizaciones sin fin de lucro que previamente tuvieran firmado un convenio con la Universidade da Coruña.

\section{RESULTADOS}

81 alumnos matriculados en la asignatura de Dirección Estratégica y Política empresarial I del grado de ADE se implicaron voluntariamente en este proyecto. Las Organizaciones No 
Gubernamentales (ONGs) analizadas, a las que previamente se había solicitado su colaboración formal a través de la firma de un convenio con la Universidade da Coruña, fueron las siguientes:

- Gatocan: Es una asociación protectora de animales de A Coruña, que lleva en funcionamiento desde el año 2006. Se encargan de recoger y dar refugio a perros y gatos abandonados, procurando que no se encuentren hacinados dentro de las instalaciones y que cuenten con espacios amplios de recreo. Gracias a esta labor, han sido declarados en 2010 Asociación de Utilidad Pública por la Xunta de Galicia.

- 0 Legado de Max: Asociación protectora de animales domésticos abandonados, ubicada en Carballo, A Coruña. Su principal objetivo es buscar un hogar digno a cada uno de los animales rescatados. Se vuelcan en el cuidado y mimo de los animales durante su estancia en el refugio, tratándolos como parte de la familia.

- Coruña Gatuna: Asociación protectora de animales especialmente centrada en prestar ayuda a gatos callejeros y colonias de gatos de la ciudad de A Coruña. Llevan a cabo diferentes acciones, que van desde el rescate, la acogida y la adopción, hasta la captura, esterilización y suelta. Ha firmado un convenio de colaboración con el Ayuntamiento de A Coruña que la acredita y autoriza al cuidado diario de estos animales.

- Residencia Padre Rubinos: Este centro ofrece atención sociosanitaria a personas mayores. Se fundamenta en la profesionalización del trabajo, equipos interdisciplinares, conocimiento científico y social, intercambio y pertenencia a asociaciones, voluntariado, redes sociales, formación e investigación.

- Teatro Colón de A Coruña: Centro de artes escénicas gestionado por el Ayuntamiento de A Coruña desde 1948.

- AfAC0: La Asociación de Familiares de enfermos de Alzheimer y otras demencias afines de A Coruña, AFACO, es una organización sin ánimo de lucro que está dirigida a mejorar la vida de las personas aquejadas de alzhéimer, así como la de sus familias, 
mediante una atención integral personalizada y de calidad contrastada. AFACO cuenta con un equipo técnico interdisciplinar especializado, con años de experiencia en la atención y el cuidado de personas con demencia.

- Equus Zebra: Desde el año 2000 la ONG Equus Zebra trabaja con la misión de integrar a personas en riesgo de exclusión social. Todo de manera cercana, flexible y comprometida con los valores de interculturalidad, justicia y solidaridad, a través de aportaciones públicas, privadas y gracias a la colaboración de personas voluntarias.

- ADEGA (Asociación de defensa ecológica de Galicia): La Asociación para a Defensa Ecolóxica de Galiza (ADEGA) es una asociación ecologista democrática, independiente y sin ánimo de lucro que trabaja en defensa del medio ambiente en Galicia.

- Centro Pai Menni: Ofrece una atención integral a personas con discapacidad intelectual proporcionándoles una intervención multidisciplinar y personalizada, teniendo en cuenta sus necesidades, capacidades y preferencias. Cuenta con una residencia para personas con grandes necesidades de apoyo, residencia para adultos con discapacidad, viviendas tuteladas, servicio de respiro familiar, centro de Día y Centro ocupacional.

- Asociación Parkinson Galicia: La Asociación Parkinson Galicia-Coruña fue fundada en 1996 por un grupo de enfermos y familiares. Desde entonces, trabaja para apoyar y mejorar la calidad de vida de las personas con la enfermedad de Parkinson y las de sus familias, poniendo a su disposición los recursos necesarios, como son: información y asesoramiento, terapias rehabilitadoras específicas y asequibles y sensibilización a la sociedad.

- Banco de Alimentos Rías Altas: Su objetivo es hacer llegar alimentos a las personas que lo necesitan, a través de los almacenes de distribución ubicados en A Coruña, Ferrol y Santiago.

- Escola infantil Padre Rubinos: Centro educativo de apoyo para niños de 0 a 3 años. 
- Asociación de persoas xordas de Ferrol: La Asociación de Persoas Sordas de Ferrolterra (AXF) nace en 1972 como punto de encuentro de las Personas Sordas de la comarca de Ferrolterra. Actualmente fomenta la inclusión social y la igualdad de las Personas Sordas a través de su empoderamiento personal, la eliminación de las barreras de comunicación y todas aquellas acciones que aseguran su pleno desarrollo como miembros activos de la sociedad.

- SOS Racismo Galicia: Los principales fines que persigue esta entidad sin ánimo de lucro son: i) luchar contra cualquier forma de discriminación por razón de color de piel, de origen, de cultura o religión para lograr una sociedad justa y libre de prejuicios y estereotipos; ii) difundir valores, actitudes y prácticas para que la población reconozca, acepte y normalice el carácter pluricultural y multirracial de la sociedad gallega, y valore positivamente la riqueza socioeconómica que aporta la diversidad y la interculturalidad y iii) favorecer la integración sociolaboral del colectivo inmigrante residente en Galicia

- Grupo Naturista Hábitat: Asociación orientada a trabajar por el estudio y la conservación de los espacios naturales y de la biodiversidad de Galicia, desde un enfoque naturalista bajo criterios científicos. Hábitat ha contribuido de manera clave a la protección de numerosos espacios naturales ahora ya emblemáticos, como la laguna de Baldaio y las Fragas do Eume. Además de su trabajo conservacionista, Hábitat organiza numerosas excursiones, cursos y charlas de divulgación de la naturaleza, dirigidos al público en general, bajo la premisa de que difícilmente se pode proteger lo que no se conoce.

- IES Universidad Laboral: El centro empieza su actividad en octubre de 1964 con el nombre de Universidad Laboral "Crucero Baleares", con dependencia del Ministerio de Trabajo y subvencionado por las Mutualidades Laborales. La orientación profesional preferente era Marítimo-pesquera, y los estudiantes procedían de toda España. Con el tiempo, la orientación profesional de Marítimo-Pesquera se amplió a otras familias 
profesionales (Electricidad, Frío y Climatización, Electrónica de Comunicaciones e Calderería), BUP, COU, estudios de la UNED y enseñanza profesional de adultos, para incorporarse más tarde a los nuevos proyectos educativos diseñados por el MEC (Bachillerato, FP específica, Educación permanente de adultos (EPA) y Programas de Garantía Social).

Siguiendo el enfoque recomendado por la Guía de Boas Prácticas de Aprendizaxe e Servizo publicado por la Universidade da Coruña en 2019, presentamos a continuación la ficha utilizada para documentar cada uno de los casos de estudio propuestos. Dado que el trabajo de los alumnos continúa durante este curso académico, sólo podemos presentar los resultados esperados, a la espera de evaluar los conseguidos a final de curso.

\section{Objetivos}

Desarrollar el análisis estratégico de una organización sin fin de lucro e identificar su formulación estratégica y posibles vectores de crecimiento que incrementen su supervivencia en el futuro.

\section{Aprendizaje}

Los estudiantes trabajarán las competencias objetivo de la disciplina de Dirección Estratégica y Política Empresarial I detalladas en la Tabla 2, aplicando el marco conceptual aprendido en el aula a una problemática empresarial real.

- Participantes: 81 estudiantes

- Destinatarios: entidades sin fin de lucro que hayan firmado previamente un convenio con la UDC

- Entidades colaboradoras: 16 entidades sin ánimo de lucro localizadas en el entorno próximo a la universidad

\section{Servicio}

Los estudiantes asumen el compromiso de entregar a los responsables de la ONG objeto de estudio el informe final, evaluado y corregido por el profesor, para que puedan utilizarlo como herramienta de mejora empresarial en su organización. 


\section{Desarrollo}

- Primera fase: contacto de los profesores con las entidades dispuestas a colaborar en el proyecto, y gestión de la firma del convenio

- Segunda fase: propuesta a los alumnos y selección de organizaciones objeto de estudio

- Tercera fase: búsqueda documental y contacto con la entidad

- Cuarta fase: aplicación de herramientas de análisis y propuesta de mejora

- Quinta fase: evaluación del informe y entrega del mismo a la entidad.

\section{Evaluación}

Está previsto que los estudiantes presenten un primer borrador del análisis y luego hagan las mejoras propuestas por el profesor a partir de la evaluación y sesiones de tutorías planificadas a tal efecto. El informe final se presentará al final del cuatrimestre y se defenderá delante de la clase. La nota del análisis (media de la nota del informe y la nota de defensa) supone un 30\% de la nota final de la asignatura de Dirección Estratégica y Política Empresarial I.

\section{Resultados}

Está previsto evaluar la satisfacción de estudiantes y entidades sin fin de lucro al final del proyecto, en junio de 2020, para obtener información sobre la utilidad de esta iniciativa y posibles mejoras a realizar.

\section{Información}

Los estudiantes podrán consultar la documentación que se maneja en la asignatura de Dirección Estratégica y Política Empresarial I, así como los datos de las entidades sin fin de lucro que figuren en las bases de datos de la Xunta y el Instituto Galego de Estadística. Así mismo, mantendrán entrevistas personales con los representantes de las entidades para obtener los datos necesarios para su análisis. 


\section{CONCLUSIONES}

A través de esta experiencia se pretende implicar a los estudiantes en su entorno y aumentar la motivación por el aprendizaje, al mostrarles una conexión más directa entre lo aprendido en clase y su aplicación a situaciones reales, mejorando las competencias profesionales establecidas en los programas de grado y de la asignatura. La orientación del proyecto aprendizaje-servicio en la asignatura de Dirección Estratégica y Política Empresarial I permite que alumnos con un perfil académico de Economía y Empresa puedan aplicar sus conocimientos para favorecer la supervivencia y el crecimiento de organizaciones menos estudiadas en el ámbito económico, como son las entidades sin fin de lucro.

En el futuro los retos pendientes son tres: la medición de inserción laboral de los estudiantes gracias al contacto que han realizado con esta experiencia, la identificación de best practices entre las entidades objeto de estudio y la formulación estratégica y el diseño de nuevas herramientas de transferencia del conocimiento adquirido a través de estos estudios entre los estudiantes del mismo grupo, distintos grupos y distintas promociones.

\section{REFERENCIAS}

Universidade da Coruña, Facultad de Economía y Empresa (2008). Memoria general del título de grado en Administración y Dirección de Empresas (http://www.economicas.udc.es/subido/Paginas\%20simples/pag20081125132922/Gra do_ADE_Aprobado_XF.pdf)

Villarreal, 0. y Landeta, J. (2010). El estudio de casos como metodología de investigación científica en dirección y economía de la empresa. Una aplicación a la internacionalización. Investigaciones Europeas de Dirección y Economía de la Empresa, vol. 16 (3), pp. 31-52.

Villarreal, 0. (2011). Cooperación en la internacionalización. Estudio temático de casos Innobasque. Agencia vasca de innovación.

Yin, R.K. (1994). Case Study Research. Design and Methods, Applied Social Research Methods Series, Vol. 5, Second Edition, Sage Publications, London. 